\title{
Culture écrite de l'Antiquité tardive et papyrologie byzantine
}

Jean-Luc Fournet

\section{(2) OpenEdition}

Édition électronique

URL : https://journals.openedition.org/annuaire-cdf/15368

DOI : $10.4000 /$ annuaire-cdf.15368

ISBN : 978-2-7226-0572-5

ISSN : 2109-9227

Éditeur

Collège de France

Édition imprimée

Date de publication : 30 décembre 2020

Pagination : 269-292

ISBN : 978-2-7226-0516-9

ISSN : 0069-5580

\section{Référence électronique}

Jean-Luc Fournet, «Culture écrite de l'Antiquité tardive et papyrologie byzantine », L'annuaire du Collège de France [En ligne], 118 | 2020, mis en ligne le 01 avril 2021, consulté le 22 août 2022. URL : http:// journals.openedition.org/annuaire-cdf/15368; DOI : https://doi.org/10.4000/annuaire-cdf.15368 


\title{
CULTURE ÉCRITE DE L'ANTIQUITÉ TARDIVE ET PAPYROLOGIE BYZANTINE
}

\author{
Jean-Luc FOURNET \\ Professeur au Collège de France
}

Mots-clés : papyrologie, Égypte, grec, copte, Antiquité tardive, multilinguisme

La série de cours « Babel sur le Nil : multilinguisme et multiculturalisme dans l'Égypte de l'Antiquité tardive (III) » est disponible, en audio et en vidéo, sur le site internet du Collège de France (https://www.college-de-france.fr/site/jean-luc-fournet/course-20172018.htm) ainsi que les colloques «Polygraphisme: écritures en coexistence et en interférence » (https://www.college-de-france.fr/site/jean-luc-fournet/symposium-20172018 _ 1.htm) et « Horapollon : hellénisme et hiéroglyphes dans l'Antiquité tardive » (https://www.college-de-france.fr/site/jean-luc-fournet/symposium-2017-2018.htm).

\section{ENSEIGNEMENT}

COURS - BABEL SUR LE NIL : MULTILINGUISME ET MULTICULTURALISME DANS L'ÉGYPTE DE L'ANTIQUITÉ TARDIVE (III). L'ÉGYPTIEN FACE AU GREC (SUITE ET FIN)

\section{Introduction}

Après avoir suivi, l'an dernier, l'émergence, au III $^{\mathrm{e}}$ siècle après J.-C., d'une nouvelle forme écrite de l'égyptien, le copte, et scruté les milieux dans lesquels ce dernier s'est développé ainsi que les modalités de sa diffusion, les cours de cette année se sont fixé comme objectif d'examiner la façon dont le copte, indissociable du christianisme, a cohabité avec le grec, langue officielle depuis la conquête d'Alexandre. Cette cohabitation fut tout sauf statique et les domaines de spécialisation qu'occupèrent copte et grec évoluèrent très sensiblement. $\mathrm{Si}$, dans un premier temps, l'usage du copte s'est restreint à la communication d'ordre privé et/ou religieux (correspondances entre particuliers ou entre membres de communautés monastiques) tandis que le grec avait le monopole de l'écrit 
administratif et juridique ainsi que celui de la création littéraire, la situation change au cours $\mathrm{du} \mathrm{VI}^{\mathrm{e}}$ siècle. Le copte commence alors à pénétrer les domaines qui lui étaient jusqu'ici fermés : il devient un idiome de production littéraire à grande échelle ; il se fait peu à peu une place dans la liturgie ; il acquiert le statut de langue juridique apte à la rédaction des transactions réglementées entre particuliers ; il finit même par être utilisé dans les rapports entre pouvoir et administrés. Les raisons de sa stagnation durant les presque trois premiers siècles de son histoire (à laquelle on trouve des parallèles dans d'autres société hellénophones du Proche-Orient) et de son émancipation croissante à partir de 550 méritent d'être dégagées. Derrière la question des langues et de leurs relations concurrentielles, ce sont les profondes mutations culturelles, religieuses et politiques que connaît la société de l'Antiquité tardive qui sont en jeu. Le multilinguisme est l'une des clés de la compréhension de ces évolutions.

\section{Cours 1 - L'usage du copte du $\mathrm{IV}^{\mathrm{e}}$ au $\mathrm{VI}^{\mathrm{e}}$ siècle : qu'écrit-on en copte plutôt qu'en grec?}

Le cours de l'an dernier s'était conclu sur un examen des divers critères permettant de mieux dégager le profil des coptographes par rapport aux hellénographes (critères religieux : païens versus chrétiens, chalcédoniens versus anti-chalcédoniens, clercs versus moines; critères socio-économiques). Ces critères s'avérant inexacts ou insuffisants, j'avais conclu que c'était celui de la fonction de l'écrit qui était le plus à même de rendre compte de la spécialisation entre copte et grec et avais réservé son étude pour cette année. Cette spécialisation est évidemment conditionnée par des normes sociales et elle se conforme aux attentes des individus, et c'est en cela qu'elle prend une dimension socioculturelle qui dépasse le niveau de simples pratiques langagières individuelles. En outre, elle se modifie avec le temps puisque le profil de la documentation dans les deux langues fait apparaître de nouvelles tendances à partir du milieu du $\mathrm{VI}^{\mathrm{e}}$ siècle.

Dans le domaine de l'écrit quotidien, celui des documents - qui semblent le plus à même de nous donner l'image la moins biaisée possible de la pratique orale et quotidienne des individus -, le copte est employé entre la fin du $\mathrm{III}^{\mathrm{e}}$ et le milieu du $\mathrm{VI}^{\mathrm{e}}$ siècle exclusivement pour la rédaction des lettres privées. On n'y a jamais recours pour les contrats (documents juridiques), les pétitions (documents judiciaires), les reçus d'impôt ou autres notifications administratives, autant de types de documents qui font la diversité et la richesse des sources papyrologiques grecques et qui ne se rencontrent, dans les archives bilingues, que dans leur composante grecque. Se dessine ainsi une scission très claire de la documentation établie sur une répartition linguistique : le copte est la langue des écrits de la sphère privée, le grec celle de l'écrit sous toutes ses formes, ressortissant aussi bien à la sphère privée que publique ou juridique; le copte se cantonne à l'écrit non informel tandis que le grec a l'apanage de l'écrit formel, celui qui est réglementé par la norme juridique ou les institutions administratives.

Les exceptions à cette répartition tranchée ont été examinées une à une (notamment des textes des années 520-550 provenant du nome Hermopolite) pour être invalidées. Seul le reçu de prêt en copte conservé par un papyrus de Kellis du IV siècle (P.Kell. VII 123) peut constituer une vraie anomalie, et encore celle-ci est-elle atténuée par sa forme épistolaire qui en fait un engagement très informel rédigé par un scribe peu au fait des formes juridiques. Il montre en tout cas qu'on a pu sporadiquement 
essayer d'utiliser le copte à d'autres fins que la communication informelle de type épistolaire. Mais le seul fait que nos Kellites, désireux d'émanciper le copte de sa seule fonction d'échange épistolaire en l'employant à des fins juridiques, ont renoncé à calquer en copte les documents juridiques grecs contemporains et se sont accommodés du moule épistolaire témoigne de la conscience qu'ils avaient d'une incompatibilité entre copte et instruments juridiques. Aussi peut-on voir dans cet unicum l'indice d'une acceptation intégrée au niveau collectif de l'incapacité du copte à pouvoir être une langue du droit.

\section{Cours 2 - La place marginale du copte dans la culture écrite}

L'invisibilité du copte dans les documents relevant de la sphère publique pendant les trois premiers siècles de son histoire a aussi son pendant dans l'espace public. Les inscriptions monumentales sont exclusivement en grec jusqu'au $\mathrm{VI}^{\mathrm{e}}$ siècle. Même les inscriptions dédicatoires d'église sont en grec, langue officielle de l'Église. Les premières inscriptions coptes relèvent du domaine privé : ce sont les épitaphes. Et, même s'il est vrai qu'elles sont difficilement datables en l'absence presque systématique d'une date absolue, elles semblent rares au $\mathrm{VI}^{\mathrm{e}}$ siècle, encore dépendantes du grec pour certaines parties. Même dans l'espace privé, l'épigraphie répugnait à faire place au copte comme en témoigne l'instrumentum domesticum, notamment la vaisselle d'apparat sur laquelle il était d'usage d'apposer des inscriptions relatives au propriétaire ou des dédicaces à des saints personnages, voire des maximes. Par exemple, toutes les cuillers inscrites d'Égypte - qui sont en train de faire l'objet d'un catalogue par Dominique Bénazeth et moi-même - sont pourvues d'inscriptions en grec. Du fait de leur caractère ostentatoire qui les destine à être lues par des personnes extérieures au cercle strictement familial, elles nous instruisent sur les choix culturels que les commanditaires souhaitaient afficher. Le copte n'y avait pas sa place.

Tandis que, dans le domaine documentaire, il n'occupe que le niveau de la communication privée, le copte ne connaît également, dans le domaine littéraire, qu'une place restreinte durant la première phase de son histoire : celle de la littérature de reproduction avec les nombreuses traductions de textes chrétiens, principalement bibliques (y compris des apocryphes de l'Ancien et du Nouveau Testament), mais aussi patristiques ou homilétiques - sans compter les traductions d'écrits gnostiques ou manichéens connus grâce aux découvertes de Nag Hammadi pour les premiers ou de Medinet-Madi ou de Kellis pour les seconds. Mais, à une exception près sur laquelle je reviendrai - mais qui n'est pas révélatrice d'une situation collective -, il peine encore à s'imposer comme médium d'une littérature originale.

Le fait que le copte fut cantonné à la copie des textes littéraires et à la rédaction des lettres privées nous a semblé d'ailleurs rendre compte d'un des traits les plus saillants de l'écriture copte documentaire : sa tendance à la non-cursivité et ses allures de majuscule, caractéristiques de la copie des papyrus littéraires ou des lettres privées. Ces deux spécificités de l'écriture copte semblent être la traduction graphique de la reconnaissance de son impropriété à un usage juridique ou administratif ; elles résultent d'une répartition sociale, consciente et volontaire entre les deux écritures, d'une digraphie socialement organisée. La preuve en est que l'écriture copte finira par adopter un style cursif lorsqu'elle cessera de n'être utilisée qu'à des fins littéraires ou épistolaires ; autrement dit quand elle sera usitée pour les textes juridiques. 


\section{Cours 3 - La situation à l'échelle de l'Empire : sources juridiques et papyrus proche-orientaux}

L'absence du copte hors du champ épistolaire, une fois établie, doit être expliquée. Pour éviter toute interprétation myope, notre enquête doit s'ouvrir à d'autres documentations et s'étendre au-delà des frontières de l'Égypte. Ce rapport de force constaté en Égypte entre langue indigène reléguée au domaine privé et langue officielle utilisée dans tous les domaines de l'écrit résulte-t-il d'une mesure du droit romain destinée à étouffer les langues vernaculaires au profit du latin ou de son Ersatz, le grec ? Ou bien est-il une situation propre à l'Égypte?

Nous n'avons pas trace d'une législation interdisant l'usage d'une langue vernaculaire. Certains juristes ont même affirmé la validité d'un usage juridique de cette dernière : Ulpien (début du $\mathrm{III}^{\mathrm{e}}$ siècle) considère que n'importe quelle langue (il cite nommément le punique ou le syriaque) peut produire une obligation à condition que les deux parties se comprennent, soit directement, soit par le truchement d'un interprète (Digeste 45, 1, 1, 6, repris par Justinien dans ses Institutes, 3, 15, 1). Il réaffirme ce principe dans le cadre du droit successoral avec les fidéicommis qui peuvent être rédigés, en plus du latin ou du grec, « en punique, gaulois ou dans la langue de n'importe quelle autre nation » (Digeste 32, 11 pr.).

Mais qu'en était-il dans la pratique ? Si les papyrus d'Égypte ne confortent pas les conclusions des juristes, en allait-il de même ailleurs? Il se trouve que nous disposons d'un désormais substantiel corpus de documents proche-orientaux, écrits dans une aire culturelle où le grec, langue officielle, côtoyait les diverses langues dérivant de l'araméen (nabatéen, palmyrénien et surtout syriaque). Or, pour le $\mathrm{III}^{\mathrm{e}}$ siècle - où apparaît le copte en Égypte -, les papyrus de Doura-Europos et de l'Euphrate attestent qu'on rédigeait des contrats dans des langues locales, qu'on n'hésitait pas à souscrire dans ces langues plutôt que de faire appel à un hypographeus ; qu'on se permettait même d'y avoir recours dans des souscriptions à un document destiné aux autorités.

Certains ont vu dans l'usage juridique de dialectes araméens une survivance d'une tradition antérieure à l'occupation romaine, ce que pourrait corroborer la disparition des contrats en langues vernaculaires après le milieu du $\mathrm{III}^{\mathrm{e}}$ siècle. Mais cette position a été contestée par d'éminents savants (G.K. Taylor, R.S. Bagnall, H.M. Cotton) qui ont souligné la vitalité de la pratique juridique en araméen malgré les aléas de notre documentation. On peut avancer comme preuves de la visibilité de ces langues vernaculaires bien au-delà du $\mathrm{III}^{\mathrm{e}}$ siècle les inscriptions monumentales syriaques qui, à compter du $\mathrm{IV}^{\mathrm{e}}$ siècle, investissent l'espace public (contrastant avec ce qui se passe en Égypte) ou les souscriptions syriaques apposées par certains évêques dans des documents officiels des conciles oecuméniques des $\mathrm{V}^{\mathrm{e}}-\mathrm{VI}^{\mathrm{e}}$ siècles alors même que le copte n'y est jamais utilisé.

Il faut bien se résoudre à penser que, contrairement à ce qui se passe ailleurs avec d'autres langues locales des provinces de l'Empire romain d'Orient, le copte ne s'est pas vu conférer une reconnaissance officielle pendant les trois premiers siècles de son histoire. L'Égypte a donc bien été, en matière linguistique, sinon une anomalie, du moins une exception. Il reste à élucider les raisons de cette position singulière du copte. 


\section{Cours 4 - L'obstacle du multidialectalisme}

Il faut commencer par se demander si les causes de la marginalisation du copte ne tiennent pas à des raisons internes à cette langue, en l'occurrence à son multidialectalisme qui l'oppose d'emblée au grec. Contrairement à ce que le terme « copte » pourrait nous faire croire, il n'y a pas une langue copte, mais une nébuleuse de variations dialectales qui se cristallisent en quelques grands dialectes. Cette variété linguistique, qui oscille entre différenciation phonologique, voire lexicale, et variabilité orthographique, pourrait avoir été un frein au développement du copte en tant que langue également compréhensible par tous et donc utilisable dans des actes administratifs ou juridiques se devant d'être intelligibles sur la totalité du territoire égyptien. Certes cette volatilité dialectale a été quelque peu contrebalancée par le développement de dialectes supra-régionaux comme le sahidique pour la vallée du Nil, selon une dynamique de standardisation qui, dès le IV siècle, aurait marginalisé les autres dialectes au profit des grandes langues véhiculaires. Mais même ceux-ci n'en sont pas moins des langues artificielles en décalage avec les langues parlées au quotidien. Artifice pour artifice, le grec pouvait servir de pis-aller : quitte à avoir recours pour la rédaction de documents réglementés à une langue non utilisée naturellement, le grec faisait en effet fort bien l'affaire.

\section{Le poids inhibant du grec et le long processus d'autonomisation : le parallèle de la littérature}

Le handicap du multidialectalisme a été amplifié par la position surplombante du grec, langue de l'administration depuis le IV siècle avant J.-C. et surtout langue qui avait su s'adapter au droit romain depuis la conquête romaine et particulièrement depuis la Constitution antoninienne (212). Cette suprématie du grec n'a pas été sans inhiber le développement du copte, qui, de formation récente, n'avait pas encore pu mener à bien le long travail d'adaptation et d'autonomisation nécessaire pour devenir une langue capable de remplir des fonctions juridico-administratives. Il ne s'agissait pas seulement de faire évoluer un patrimoine linguistique préexistant, mais, à la suite de la solution de continuité entre la fin du démotique et l'émergence du copte, de recréer ce patrimoine en adaptant une langue fortement renouvelée à l'univers complexe et pour elle si étranger du droit romain.

Ce processus d'autonomisation n'est d'ailleurs pas propre à l'écrit juridicoadministratif ; on en suit aussi la trace dans le domaine de la littérature. Quoique notre connaissance de celle-ci soit encore incertaine et quelque peu obérée par la lancinante incertitude du véritable pedigree linguistique d'œuvres transmises en copte mais non nécessairement composées dans cet idiome, on peut être en effet tenté de mettre en parallèle l'écart qui sépare les premiers essais d'utilisation du copte documentaire (pour les lettres) des premiers documents juridiques (seconde moitié $\mathrm{du} \mathrm{VI}^{\mathrm{e}}$ siècle) avec l'intervalle que l'on observe, dans le domaine de la littérature, entre les premiers témoins du copte littéraire (glossaires, traductions) et le développement d'une littérature copte originale, surtout à partir de la fin du $\mathrm{VI}^{\mathrm{e}}$ siècle. Shénouté a beau avoir été, dès la fin du IV siècle, le premier grand auteur original de la littérature copte (et peut-être le plus grand), il faut en effet attendre l'épiscopat de Damien (578-605) pour assister au véritable épanouissement d'une production littéraire de langue copte qui ne soit pas le fait d'une personnalité d'exception mais participe d'un mouvement à grande échelle. On a d'ailleurs fait remarquer que la quantité des œuvres allait de pair avec leur qualité. Tito Orlandi, 
soulignant le saut qualitatif que ce mouvement représente dans l'histoire de la langue copte, n'hésitait pas à parler de «progrès naturel dans la langue et [de] son indépendance croissante par rapport au grec ». Il en est allé de même avec l'écrit documentaire : il a fallu du temps pour que les Égyptiens pensent leur langue non comme un simple médium de communication privée mais comme une «institution » cristallisant un certain nombre de valeurs partagées collectivement et capable d'avoir dans tous les domaines autant d'autorité et de prestige que le grec.

\section{Cours 5 - L’Église elle aussi hellénophone}

En plus d'être la langue de l'État, le grec fut aussi celle de l'Église d'Égypte depuis ses origines, ce qui acheva de conforter sa position privilégiée et contribua à l'inhibition du copte. On pourrait pourtant penser que l'Église aurait eu à cœur d'encourager ce dernier, lié au développement du christianisme. Mais elle était née avant l'invention du copte et avait dû se construire et s'affirmer en ayant recours au grec, qui se trouvait, en outre, être la langue des livres sacrés sur lesquels elle appuyait sa doctrine et celle des ouvrages de théologie écrits par les pères de l'Église de la partie orientale de l'Empire à laquelle appartenait l'Égypte. Le monolinguisme de l'Église, dans son organisation et son administration, s'affichait jusque dans la liturgie, touchant ainsi les fidèles à travers la langue qu'ils se voyaient obligés de pratiquer durant les offices. La langue liturgique de l'Église d'Égypte resta en effet pendant longtemps le grec, et cela ne contribua pas peu à reléguer le copte au rang de low language. Si les prières sont les premiers types de textes liturgiques à avoir fait place au copte - et encore faut-il attendre les $\mathrm{VI}^{\mathrm{e}}-\mathrm{VII}{ }^{\mathrm{e}}$ siècles -, les hymnes (parties chantées) et plus encore les acclamations (dialogues codifiés entre les diverses parties prenantes de la liturgie) résistèrent à la pénétration du copte au moins jusqu'aux VIII ${ }^{\mathrm{e}}-\mathrm{IX}^{\mathrm{e}}$ siècles. Ce sont en effet les parties les plus formelles de la liturgie, pour lesquelles on s'est senti obligé de recourir au high language, à la langue de prestige, malgré les évolutions linguistiques naturelles de la société.

Le maintien du grec comme langue par défaut de la liturgie ne fut évidemment pas sans rendre la position des fidèles de plus en plus inconfortable. L'écart entre l'usage liturgique et leur véritable maîtrise linguistique se creusait toujours plus jusqu'à un point de rupture atteint au $\mathrm{VIII}^{\mathrm{e}}$ siècle. Si cet écart était neutralisé à l'oral par la force de l'habitude et la capacité des fidèles à reproduire mimétiquement la sonorité de paroles apprises par cœur et parfois par le recours à des traductions - ainsi que par la présence d'interprètes attestée pour l'Égypte par Sérapion de Thmouis (IV siècle) -, il n'en allait pas de même à l'écrit. Et c'est ainsi qu'on voit se multiplier des hymnaires ou autres recueils liturgiques à ce point truffés de fautes que le grec en devient méconnaissable.

\section{Cours 6 - Le poids écrasant du passé : la marginalisation institutionnelle de l'égyptien}

L'égyptien n'a pas toujours été aussi dépendant du grec et relégué aux lettres privées. Le démotique a été pendant longtemps usité pour d'autres types de documents (contrats, documents fiscaux ou judiciaires) avant qu'une politique, esquissée par les Lagides dès le milieu du $\mathrm{II}^{\mathrm{e}}$ siècle avant J.-C. et accentuée par les Romains, ne vise à en restreindre l'usage dans le domaine juridico-administratif. Il finit par s'étioler à partir du I ${ }^{\mathrm{er}}$ siècle puis disparaître, laissant les Égyptiens dans une 
situation d' «agraphie » collective (voir cours 2 de l'an dernier). Cette rupture de continuité pesa lourdement sur le développement du copte, qui devait à la fois retrouver sa place dans une culture écrite désormais totalement occupée par le grec mais aussi reconquérir un statut officiel qui avait permis à l'égyptien de jadis de jouer un rôle juridique et administratif.

\section{Le copte victime de ses origines}

Mais en fin de compte, la raison de la marginalisation du copte ne tient-elle pas aux coptographes eux-mêmes ? Ont-ils vraiment cherché, dans un premier temps, à conquérir pour leur langue un statut officiel ? Nous avons vu l'an dernier combien le copte était tributaire du grec (pour son alphabet, son lexique, la diplomatique de ses documents) et combien ses inventeurs et promoteurs étaient profondément hellénisés. Suffisamment en tout cas pour continuer à s'accommoder sans difficultés du cadre traditionnel et pour ne pas chercher à revendiquer un usage plus large du copte dans la société. La fonction première qui était conférée à cet idiome naissant était avant tout une fonction sacrée : celle de fournir une traduction en égyptien de leurs saints livres à cette nouvelle classe montante de chrétiens qui commençaient à jouer un rôle social et politique de plus en plus marqué. Un usage séculier, en dehors de la communication privée, n'a pas dû s'imposer à eux comme une nécessité. Le recours au copte pour les actes juridiques s'inscrivant jusqu'ici dans un cadre grec devait leur sembler, à eux bilingues, parfaitement inutile, d'autant plus que ces actes étaient rédigés par des professionnels accomplis en grec, maîtrisant les arcanes du droit romain, dans un contexte étatique verrouillé par l'usage exclusif du grec. C'est donc peut-être dans les liens si forts qu'il a entretenus dès ses origines avec l'hellénisme et dans l'hellénisation si profonde de ses inventeurs et des premières générations de ses utilisateurs qu'il faut chercher la raison de l'inhibition qui a maintenu le copte si longtemps hors de la sphère publique et a détourné ses utilisateurs du désir de l'imposer comme un concurrent possible du grec dans les domaines de l'écrit réglementé.

Mais le copte a «échappé » à ses premiers utilisateurs et s'est dans un second temps répandu dans d'autres milieux. Sa sociologie s'est modifiée en même temps que le cadre culturel et institutionnel commença à se transformer, rendant possible, un jour ou l'autre, un usage officiel du copte.

\section{Cours 7 - L'apparition des premiers documents juridiques en copte}

On aurait pu s'attendre à ce que l'émancipation du copte soit une des conséquences de la conquête arabo-musulmane (641-642) qui coupa le lien entre l'Égypte et l'État byzantin hellénophone et rendait ainsi possible un usage plus visible du copte dans la société avant que celle-ci ne s'arabise. C'est pourtant un siècle avant que ce processus s'amorça. Même s'ils sont peu nombreux (une quinzaine seulement si l'on se limite à ceux datés ou assez précisément datables), les documents non épistolaires coptes antérieurs à la Conquête témoignent d'une mutation sociolinguistique encore sousétudiée.

Les trois plus anciens sont d'un genre un peu particulier puisqu'il ne s'agit pas d'actes juridiques stricto sensu, mais de procès-verbaux d'arbitrage privé. Les autres sont de vrais actes juridiques (contrats de location, de mariage, ventes à terme de produits, reconnaissances de dette, testaments) qui, quoique non écrits par des notaires, calquent la diplomatique des actes notariaux grecs. Ils actent - à trois 
exceptions près - des transactions de médiocre importance (location, prêts). Il faudra attendre la conquête arabe pour qu'apparaissent vraiment les premières ventes immobilières ou autres documents concernant des transferts de propriétés. On a clairement l'impression d'une phase transitoire. Quant à leurs rédacteurs, loin d'être non hellénophones comme on aurait pu le penser a priori, ce sont de véritables bilingues, au moins pour ceux dont le profil peut être reconstitué (Dioscore d'Aphrodité, Paul fils de Megas et Daniel fils d'Hêrakleidês).

Tous nos documents proviennent de la province de Thébaïde, c'est-à-dire du sud de la Haute-Égypte. Ce n'est pas un hasard : la province de Thébaïde, depuis toujours éloignée du centre névralgique de l'Égypte, Alexandrie la grecque, et, en dehors de ses grands centres, moins profondément assujettie à son influence en matière institutionnelle, religieuse et culturelle que la Basse-Égypte ou l'Arcadie, a pu être le terreau d'une affirmation culturelle qui s'est, entre autres, exprimée, au niveau de la langue, par un recours plus rapide et plus intensif au copte.

Par ailleurs, en dépit de leur relative rareté, on constate que le nombre de ces documents augmente dans les années 600-610 et se maintient à peu près au même niveau dans la décennie 620-630. Et c'est durant ces deux décennies qu'ont été établies les trois transactions d'une valeur bien supérieure aux autres documents. Or ces deux décennies correspondent à deux époques troublées de l'histoire de l'Égypte : la première, inaugurée par le renversement et l'assassinat de Maurice par Phocas (602), fut une période de turbulences. Quant à la seconde, elle est marquée par l'occupation sassanide (619-629) qui a soustrait l'Égypte à l'autorité byzantine. L'instabilité politique, en affaiblissant l'appareil étatique, et surtout, durant la période sassanide, la rupture avec l'État byzantin hellénophone, en relâchant la contrainte que faisait peser le prestige du grec, ont dû concourir à agrandir la brèche qui avait été timidement entrouverte au siècle précédent en incitant la population qui le souhaitait à avoir un usage plus intensif du copte.

En fin de compte, les approches géographique et chronologique ont ceci en commun qu'elles orientent vers une explication qui mettrait le développement des documents juridiques coptes sur le compte d'un recul, d'un affaiblissement ou d'un défaut de l'État et donc d'un allègement de la pression qu'il pouvait faire peser en matière de contraintes linguistiques.

\section{Cours 8 - La culture grecque en perte de vitesse}

La montée du document juridique en copte s'inscrit plus généralement dans un contexte de déclin du grec et de l'hellénisme en Égypte dont témoigne à sa façon la documentation papyrologique (figure 1). Le développement des premiers textes juridiques coptes dans les années 550-642 correspond à une période qui voit l'accélération de la montée des documents coptes en général (qui deviennent majoritaires entre les $\mathrm{VII}^{\mathrm{e}}$ et $\mathrm{VIII}^{\mathrm{e}}$ siècles) et le renversement du rapport entre textes littéraires grecs et coptes. Étant donné le retard de la papyrologie copte, il est probable que les deux courbes des documents grecs et coptes devraient se croiser plus tôt, entre le $\mathrm{VI}^{\mathrm{e}}$ et le $\mathrm{VII}^{\mathrm{e}}$ siècle. C'est donc durant cette période que se produisit un changement de paradigme linguistico-culturel. La culture grecque continue certes à être un modèle, un étalon, mais dans des milieux qui se rétrécissent, quoique surreprésentés dans la documentation. C'est dans le domaine religieux qu'elle va survivre le mieux, en se renouvelant à la faveur d'un processus qui synthétise hellénisme et christianisme. Mais c'est là aussi qu'elle est le plus à même d'être 


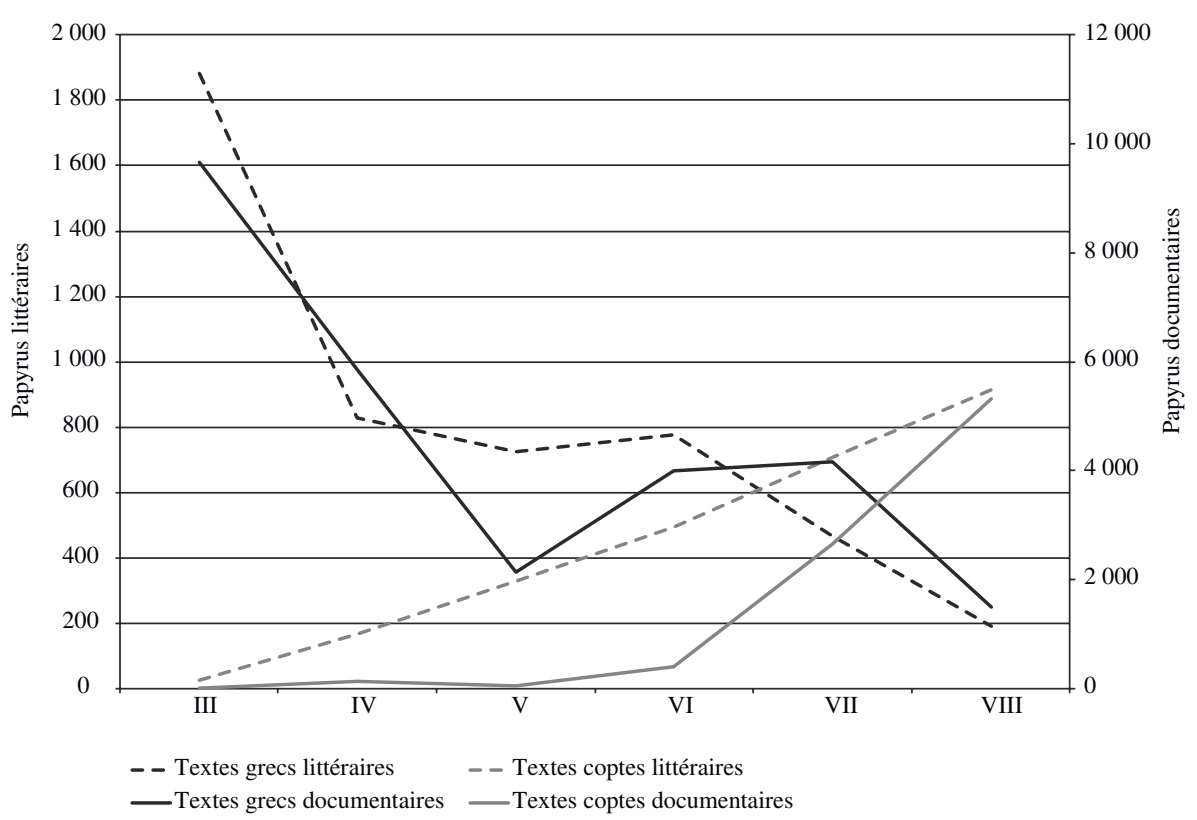

Figure 1 - Comparaison entre le nombre de papyrus documentaires et littéraires en grec et en copte.

concurrencée par le copte, d'autant que certains milieux monastiques affichent un rejet de la paideia grecque.

\section{Une crise des institutions judiciaires ? Une justice en copte}

Cette évolution culturelle sert de toile de fond à l'émergence du copte juridique ; elle ne lui fournit pourtant pas une explication suffisante. Dans la mesure où nos documents ont pour fonction d'affirmer les droits des individus qui y ont recours et s'inscrivent donc dans un cadre juridique et éventuellement judiciaire (en cas d'infraction), la cause première de leur apparition ne serait-elle pas à chercher dans une mutation des conditions de fonctionnement de la justice, qui se rendait alors exclusivement en grec? Or ils émergent au moment où s'effondre le nombre de pétitions, pièces maîtresses de la procédure judiciaire, et où disparaissent les procèsverbaux d'audiences judiciaires, qui constituent le complément procédural de la pétition. C'est aussi l'époque où se multiplient les arbitrages privés et se développent des procédures étatiques moins formelles. Ces phénomènes ont été diversement interprétés et articulés. La documentation et sa répartition chronologique semblent favoriser l'idée d'un processus en deux étapes, chacune ayant joué son rôle dans le développement du copte :

1) Dans un premier temps, le recours de plus en plus massif aux arbitrages privés, dès la première moitié du $\mathrm{VI}^{\mathrm{e}}$ siècle, a stimulé l'emploi du copte : le caractère plus informel des auditions arbitrales encourageait le recours à la langue qui était 
la plus naturelle, le copte pour les non-hellénophones. La mise par écrit des procèsverbaux de ces arbitrages en copte a eu une conséquence secondaire, celle de favoriser le développement d'un vocabulaire juridique et de contribuer à répandre l'idée que le copte n'était pas nécessairement condamné au seul échange épistolaire.

2) Dans un second temps (fin $\mathrm{VI}^{\mathrm{e}}$ et surtout début $\mathrm{VII}^{\mathrm{e}}$ siècle), la perte de vitesse - pour quelque raison que ce fût - de la procédure étatique normative (la procédure par pétition et son corollaire, le procès en grec devant le gouverneur), remplacée par des procédures moins formelles, a encouragé des recours en copte d'autant plus que ceux-ci étaient instruits par des fonctionnaires inférieurs, proches de la population, ce qui n'a pas manqué d'accréditer l'idée que le copte pouvait être utilisé dans un cadre étatique.

Parmi ces fonctionnaires, le defensor civitatis, le juge des petits, a joué un rôle déterminant comme en témoigne un document jusqu'ici mal interprété (SB Kopt. IV 1709, rééd. A. Delattre et J.-L. Fournet, Chronique d'Égypte 93, 2018, p. 145-165) : loin d'être une simple lettre, ce texte, datable d'avant la conquête arabe, est une pétition en copte adressée par une dame qui se plaint de ne pas avoir touché la pension alimentaire que lui doit son mari. Elle est accompagnée de la sentence du defensor en copte. Ce document apporte la preuve que l'administration judiciaire s'était finalement ouverte à la langue copte en autorisant la rédaction et l'instruction de pétitions dans cette langue.

\section{Cours 9 - Le rôle de l'Église et du monachisme dans l'officialisation du copte}

Tandis que l'État commençait à faire preuve de tolérance linguistique, d'autres milieux, depuis longtemps ardents promoteurs du copte, ont eux aussi contribué à l'émergence d'un copte « officiel » dépassant les frontières de l'échange épistolaire privé : il s'agit des moines (et, à travers eux, de l'Église), dont l'apport peut être mieux compris grâce à des documents inédits ou mal connus.

Le premier est un codex inédit de tablettes du $\mathrm{VI}^{\mathrm{e}}$ siècle du musée du Louvre, le codex Weill, déchiffré l'an dernier en séminaire (figure 2). Il comprend vingt-six reçus pour une part de nature fiscale et se rapportant au même contribuable (un certain Jacob), écrits en grec (20) ou - plus curieusement pour l'époque - en copte (6), entre autres par des moines de la « Fédération de Shénouté » (dans la région de Panopolis). Ce codex offre donc un témoignage ancien de l'utilisation du copte dans un contexte fiscal (autrement dit administratif) sous l'impulsion d'une institution monastique.

Les archives du monastère de Phoibammôn (sur la rive ouest de Thèbes) montrent avec plus de précision encore la part du monachisme dans la montée du copte et dans l'élaboration d'une langue capable de concurrencer le grec dans des usages non privés. Elles se composent, entre autres, d'un ensemble de testaments des quatre supérieurs de cet établissement, dont deux antérieurs à la conquête arabe, le testament d'Abraam de la fin des années 610 et celui de Victor de 634 (P.Mon.Phoib. Test. 1 et 2). Or le premier a été rédigé en grec quoique Abraam ne connût pas cette langue, le second en copte, illustrant le basculement qui s'est produit dans les premières décennies $\mathrm{du} \mathrm{VII}^{\mathrm{e}} \mathrm{s}$. en faveur du copte, peut-être à la suite de l'assouplissement résultant de l'occupation sassanide.

Si le cadre juridico-linguistique avait changé, la fonction du testament aussi : quand on compare les deux testaments, on est frappé par l'insertion, dans celui de Victor, d'éléments narratifs justifiant le choix de ses successeurs et motivant la 


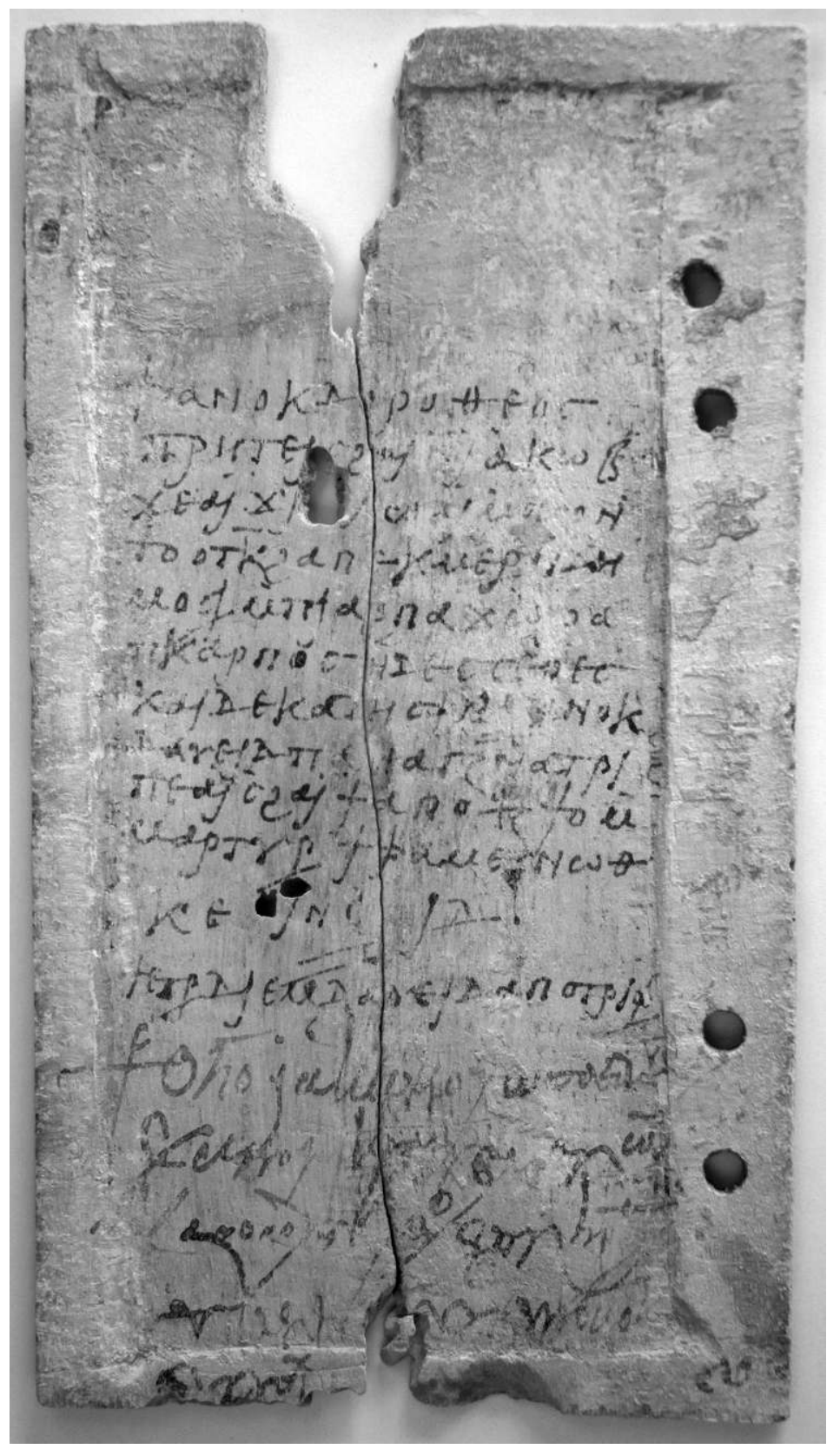

Figure 2 - Une page du codex Weill montrant un reçu copte dans la partie supérieure et grec dans la partie inférieure.

(C) Musée du Louvre, photographie infrarouge : J.-L. Fournet. 
rédaction du testament, absents de celui d'Abraam. Aussi le testament de Victor assume-t-il, outre sa fonction juridique (l'institution d'héritiers), une mission spirituelle : il véhicule un message à destination collective et à finalité édifiante et spirituelle, ce qui nécessitait qu'on s'adressât à la collectivité dans une langue compréhensible. Le testament ne pouvait donc être pleinement opératoire qu'en copte. Aussi les supérieurs de monastères ont-ils pris l'habitude de tester dans cette langue à partir du moment où les conditions juridiques le permettaient.

En plus de son testament, Abraam, nommé par Damien évêque d'Hermonthis tout en continuant à résider dans son monastère, a laissé une grande quantité de textes coptes liés à sa charge épiscopale, dont un peu moins de la moitié sont des documents non épistolaires, relevant du domaine juridique ou judiciaire (déclarations d'engagements, cautionnements, dépôts de témoignages, etc.). Ces textes - comme ceux des archives de Pisenthios de Coptos, lui aussi moine nommé évêque par Damien - sont la preuve la plus éclatante de ce que l'Église, à travers certains de ses évêques, a ressenti la nécessité de se doter d'une panoplie d'instruments juridiques, correspondant à ses missions et reconnus par l'administration, en acclimatant au copte des genres documentaires grecs par tout un travail d'adaptation typologique et lexicale. Ces prélats choisis pour leurs qualités morales qui en faisaient aux yeux de la population des sortes de holy men capables aussi de servir d'intermédiaires avec l'administration ont ainsi concouru à transférer sur une échelle plus large des pratiques issues de leurs communautés monastiques. Leurs fonctions épiscopales leur donnaient la surface nécessaire pour imposer leur langue naturelle dans des domaines écrits où le grec était encore exclusif. Ils ont contribué à l'élaboration d'un stock de types documentaires reproductibles à l'infini et au développement d'une gamme de formulaires et d'expressions juridiques empruntés au grec, mais désormais intégrés dans une diplomatique d'expression copte. À côté des quelques tentatives menées dès le $\mathrm{VI}^{\mathrm{e}}$ siècle dans la société laïque, ils ont ainsi concouru à ouvrir la voie et à «décomplexer» le copte en lui procurant sans cesse plus de ce prestige dont le grec avait le monopole.

Il est notable que ce processus soit en parfaite synchronie avec ce que nous avons constaté dans le domaine de la littérature et que ce soit certains des acteurs de cette éclosion littéraire qui contribuèrent au développement du copte documentaire (Pisenthios de Coptos). Le processus d'autonomisation du copte dans le domaine documentaire rejoint donc celui à l'œuvre dans le domaine littéraire. Cette synchronie est la confirmation d'une mutation culturelle profonde que connaît la société égyptienne à la fin $\mathrm{du} \mathrm{VI}^{\mathrm{e}}$ et au début du VII ${ }^{\mathrm{e}}$ siècle, en même temps qu'elle montre sur un plan plus méthodologique que l'écrit quotidien ne peut être conçu indépendamment de l'écrit pérenne (la littérature), tous deux relevant des mêmes tendances lourdes à l'œuvre dans la société.

COURS À L'EXTÉRIEUR - IL DOCUMENTO: UN NUOVO SGUARDO SULLA CULTURA SCRITTA DELLA TARDA ANTICHITÀ

\section{Istituto papirologico «Vitelli», Florence}

1) I cambiamenti formali del documento alla fine dell'Antichità (8 mai 2018)

2) La cristianizzazione dello scritto (15 mai 2018)

3) La 'letterarizzazione' del documento (22 mai 2018)

4) La 'documentarizzazione' della letteratura (29 mai 2018) 
I due termini, cultura e documento, sono abitualmente percepiti, se non completamente antitetici, almeno come provenienti da due diversi ambiti : queste conferenze hanno cercato di rompere questo sterile antagonismo, provando di mostrare quanto la nostra conoscenza della cultura scritta della tarda Antichità possa guadagnare nel prendere in considerazione l'ambito documentario, tanto più che quest'ultimo fu influenzato dalla cultura letteraria, con un'intensità crescente a partire dalla fine del terzo secolo, e soprattutto del quarto. Il documento è davvero un oggetto culturale a doppio titolo : (1) Esso illustra ovviamente le modalità della cultura scritta, dal momento che questa non si limita alla riproduzione dei libri o alla produzione di nuove opere, ma si realizza parimenti nell'elaborazione dei documenti. (2) $\mathrm{E}$, in maniera più inaspettata, documenta i rapporti che la società della tarda Antichità intrattiene con la cultura più specificamente letteraria, in quanto questa diventa, in misura maggiore che nelle epoche precedenti, così invadente da penetrare nell'ambito del documento, il quale finisce per diventare un buon osservatorio delle concezioni che avevano della cultura gli Antichi - e così dicendo non mi riferisco a qualche figura di uomini letterati, ma agli uomini di tutte le classi sociali.

Adottando un approccio diacronico (mostrando le rotture del documento della tarda Antichità in rapporto all'epoca romana, e le evoluzioni che il documento compie durante la fine dell'Antichità) e, allo stesso tempo, sincronico, queste conferenze hanno ricollocato i cambiamenti formali, lessicali e fraseologici che sperimenta lo scritto documentario nel contesto delle gravose tendenze all'opera nella società dell'epoca, e mostrato come questo rappresenta, a suo modo, un importante tassello del mosaico culturale della tarda Antichità.

\section{COLLOQUE - HORAPOLLON : HELLÉNISME ET HIÉROGLYPHES DANS L'ANTIQUITÉ TARDIVE}

Ce colloque, qui s'est tenu les 13 et 14 juin 2018, est le premier consacré à Horapollon, connu comme l'auteur d'un traité sur les hiéroglyphes (Hieroglyphica) le seul qui nous soit parvenu de l'Antiquité - mais qui reste une figure mystérieuse. On l'identifie à un philosophe homonyme du $V^{\mathrm{e}}$ siècle, issu d'une famille d'intellectuels païens qui tentèrent de résister au christianisme devenu religion d'État en combinant exaltation d'un hellénisme profane et défense d'une culture pharaonique en voie d'extinction (les hiéroglyphes étaient alors une écriture morte). Cette biculturalité est au cœur des Hieroglyphica : si à peu près la moitié des signes hiéroglyphiques traités par Horapollon est plus ou moins correcte, l'exégèse purement allégorique que l'auteur en donne est surtout tributaire de traditions propres à l'hellénisme tardif. Ces deux aspects de l'œuvre ont été traités lors des deux premières séances : des égyptologues spécialistes de l'écriture hiéroglyphique et de la culture égyptienne d'époque romaine ont montré en quoi le traité d'Horapollon est le reflet du dernier état de l'antique écriture hiéroglyphique et des spéculations dont elle faisait l'objet dans les milieux cléricaux égyptiens. Des spécialistes de l'Antiquité tardive ont de leur côté replacé ce traité dans la culture de cette époque, marquée par la confrontation de la pensée égyptienne et de la philosophie grecque, du paganisme agonisant et du christianisme triomphant.

Ce n'est pas le moindre apport de ce colloque que d'avoir montré que les Hieroglyphica ne sont certainement pas du philosophe Horapollon (voir les communications de G. Agosti et J.-L. Fournet) mais qu'ils sont une compilation plus 
tardive réalisée à partir de matériaux anciens (peut-être le traité sur les hiéroglyphes du philosophe Chærémon).

Depuis sa redécouverte en 1419, l'œuvre attribuée à Horapollon attisa l'intérêt pour les hiéroglyphes en marquant profondément l'iconographie de la Renaissance et la littérature emblématique. C'est ce que des spécialistes d'iconologie, de littérature et d'histoire de l'art ont contribué à mettre en lumière dans un troisième volet consacré à la postérité d'Horapollon.

Il est ressorti de ce colloque que les Hieroglyphica sont une œuvre encore entachée de zones d'ombre : une édition moderne qui revienne sur le problème de l'attribution et combine, dans un commentaire suivi, les apports de l'égyptologie et de la philologie classique est un desideratum de même que le serait une histoire de l'« horapollonisme » à l'époque moderne.

\section{Programme}

\section{L'arrière-plan égyptien des Hieroglyphica}

Andréas STAUDER (EPHE), «Les faces multiples du signe graphique égyptien »; Joachim Friedrich QUACK (Universität Heidelberg), «Les traités systématiques des Égyptiens sur les sens de leurs signes écrits »;

Sydney AUFRÈRE (CNRS-Aix-Marseille Université), «Les Hieroglyphica d'Horapollon, un héritage de la "philosophie" égyptienne au V' siècle de notre ère »; Jean WINAND (Liège université), « Horapollon ou la fin d'un parcours ».

\section{Une œuvre grecque de l'Antiquité tardive}

Jean-Luc FouRNET (Collège de France-EPHE), «Horapollon: une énigme à déchiffrer »;

Gianfranco AGOSTI (Sapienza Università di Roma), «Elementi tardoantichi nel trattato di Orapollo »;

Antonio RicciardetTo (Collège de France), «Horapollon témoin de son temps : les références aux pratiques encore en vigueur dans les Hieroglyphica ( $\mathrm{V}^{\mathrm{e}}$ siècle) »;

Arnaud ZuCKER (université Nice Sophia Antipolis), «Hieroglyphica, Physiologus, Cyranides: dénaturation ou remotivation du savoir zoologique (la parenté sémiotique/structurelle et la communauté naturaliste entre les Hieroglyphica et le Physiologus)»;

Nicola ZITO (Sorbonne Université-UMR 8167), «Les mirabilia dans les Hieroglyphica d'Horapollon : modes d'emploi »;

Mélanie Mougin (EPHE), «Le lion dans les Hieroglyphica d'Horapollon ».

\section{La postérité des Hieroglyphica}

Stéphane Rolet (université Paris 8), «Les essais hiéroglyphiques de Filippo Alberici (ca. 1507 ?) : entre Horapollon et Poliphile » ;

Mino Gabriele (Università di Udine), « Horapollo, il Polifilo, Alciato e Cesare Ripa : dal simbolismo geroglifico all'iconologia didattica »; 
Michel Hochmann (EPHE), «Horapollon, une clé pour l'interprétation de Giorgione ? »;

Romain MENINI (université Paris-Est) « Horapollon au temps de Rabelais » ;

Anna BAYDOva (EPHE), «L'illustration des Hieroglyphica d'Horapollon au $\mathrm{XVI}^{\mathrm{e}}$ siècle »;

Jean-Marc MANDOSIO (EPHE), «La représentation du personnage d'Horapollon (sa vie, son œuvre) chez les auteurs des XVI ${ }^{\mathrm{e}}$ et $\mathrm{XVII}^{\mathrm{e}}$ siècles ».

\section{COlLOQUE - PATRIMOINE PARTAGÉ OU PATRIMOINE À USAGE IDENTITAIRE}

Ce colloque, organisé à Beyrouth et Byblos, les 23 et 24 octobre 2017, avec JeanMichel Mouton (EPHE) et Jacques Paviot (UPEC) et en collaboration avec le Centre international des sciences de l'homme de Byblos (sous l'égide de l'Unesco) et la faculté des lettres et des sciences humaines de l'université Saint-Joseph de Beyrouth et avec le soutien de la Fondation Hugot du Collège de France, avait pour but de réunir des historiens, des sociologues et des conservateurs pour identifier dans l'histoire du Proche-Orient jusqu'à l'époque contemporaine les facteurs qui ont entraîné la destruction de biens patrimoniaux et qui menacent encore ce patrimoine, en s'interrogeant sur les processus qui ont conduit à la mise en péril d'un site ou d'un monument vénéré et protégé pendant des siècles. Les actes ont été publiés (cf. bibliographie du professeur).

\section{Programme}

Henri-Paul FRANCFORT (membre de l'Institut, directeur de recherches émérite au CNRS), «Mise en abyme de monuments et patrimonialisation : quelques exemples antiques de la Méditerranée à l'Asie centrale »;

Jean-Luc FouRnET (Collège de France), «Changements religieux et patrimoine cultuel : le sort des temples égyptiens durant l'Antiquité tardive »;

Jean-Michel MouTON (EPHE), «Préservation et destruction des vestiges de la ville antique de Damas à l'époque médiévale »;

Jacques PAVIOT (UPEC), «Les pèlerins occidentaux et le patrimoine religieux au Proche-Orient au Moyen Âge »;

Dominique PIERI (directeur du département scientifique Archéologie et histoire de l'Antiquité de l'Institut français du Proche-Orient, Paris I), «Le sanctuaire paléochrétien de Saint-Syméon en Syrie du Nord : un site dans la tourmente »;

Jean-Pierre BRUN (Collège de France) : «Dans le désert Oriental d'Égypte : d'un état de préservation exceptionnel à la destruction totale des sites archéologiques »;

Annie Tohmé TABET (université de Saint-Joseph), « Conséquences des destructions des monuments et des sites patrimoniaux causées par les guerres sur le patrimoine culturel immatériel : quelques exemples du Moyen-Orient»;

Hani HAYAJNEH (Yarmouk University), « The impact of tourism on the tangible and intangible cultural heritage of Petra and Wadi Ram (Southern Jordan) »;

Yves BRULEY (EPHE), «Patrimoine partagé ou identitaire ? La diplomatie française et les lieux saints de Jérusalem et Bethléem au milieu du XIX ${ }^{\mathrm{e}}$ siècle »; 
Jean-Olivier GuILHOT (inspecteur général des patrimoines au ministère de la Culture), «Légiférer sur l'archéologie : une évidence patrimoniale ou une stratégie d'appropriation territoriale ? Les priorités de la France autour de la Méditerranée (1835-1941)»;

Qahtan AL-ABEED (directeur du musée de Bassorah), «Le patrimoine religieux post-conflits confessionnels et armés : l'exemple de Bassorah (Iraq) »;

Liliane BARAKAT (université de Saint-Joseph), «Patrimoine urbain et mémoire d'une ville : Beyrouth »;

Suzy HAKIMIAN (conservateur du musée MIM, ancien conservateur du musée national de Beyrouth, université de Saint-Joseph), «Réflexions sur la sauvegarde du patrimoine à travers l'expérience du Liban ».

\section{JOURNÉE D'ÉTUDES - POLYGRAPHISME : ÉCRITURES EN COEXISTENCE ET EN INTERFÉRENCE}

Cette journée d'études, coorganisée au Collège de France le 6 avril 2018 avec Marc Smith (ENC-EPHE) et Judith Olszowy-Schlanger (EPHE), s'inscrit dans les activités du Groupe de recherches transversales en paléographie (GRTP), qui a pour vocation de développer une approche transdisciplinaire de la paléographie (voir la précédente intitulée «Type, style, main - Normes collectives, variations individuelles et évolution des écritures » qui s'est tenue au Collège de France, le 7 décembre 2016).

Cette journée proposait une réflexion sur le di-/polygraphisme individuel - pendant écrit du bi-/multilinguisme -, un phénomène constant dans les sociétés de toutes époques où coexistent divers systèmes d'écriture, souvent - mais pas toujours - liés à plusieurs langues. Outre les problèmes méthodologiques qu'il pose (comment détecter qu'une même personne écrit quand on a affaire à des systèmes d'écriture très hétérogènes ?), les divers intervenants ont cherché, chacun dans son domaine, à éclairer les conditions et modalités d'apprentissage par un même individu de plusieurs écritures ainsi que les phénomènes d'interaction ou d'interférence qui ne manquent de se produire entre elles.

\section{Programme}

Pierre-Luc ANGLES (EPHE-université d'Heidelberg), «La bilittératie grécodémotique »;

Jennifer CROMwell (université de Copenhague), « Coptic-Greek biscriptality and language policy in early Islamic Egypt »;

Michaël GUICHARD (EPHE), «Écritures en contact en Mésopotamie »;

Margherita FARINA (UMR 8167), «La relation entre digraphisme et bilinguisme dans les manuscrits syriaques. Syriaque-grec et syriaque-arabe »;

Judith OlszOwY-ScHLANGER (EPHE), «Au carrefour paléographique: le digraphisme des scribes juifs et son rôle dans le changement de l'écriture hébraïque »;

Philippe PAPIN (EPHE), «L'écriture en chinois des élites et l'écriture démotique au village : le cas du Viêt Nam »; 
Peter STOKES (EPHE), «Relations entre minuscule caroline et minuscule vernaculaire en Angleterre du XI ${ }^{\mathrm{e}}$ siècle »;

Marc SMith (ENC-EPHE), « Langues et écritures d'Occident à l'époque moderne ».

\section{RECHERCHE}

\section{TRAVAUX DE RECHERCHE}

Le multilinguisme dans l'Égypte byzantine a continué à mobiliser ma recherche tout au long de l'année en liaison avec mon enseignement. Il a été le sujet de plusieurs conférences à l'extérieur, notamment à l'université de Nanterre (« Multiculturalisme et multilinguisme dans l'Égypte gréco-romaine : les arts et les mots », le 19 décembre 2017) où j'ai présenté une tentative de démarche croisée examinant le phénomène du multiculturalisme concomitamment à travers ses deux différents modes d'expression majeurs que sont la langue et l'onomastique, d'un côté, et l'expression plastique ou visuelle, de l'autre. Malgré les limites de la confrontation qui donnent à ce travail une valeur avant tout heuristique, la combinaison des deux approches s'est avérée féconde en mettant en lumière certains mécanismes qui s'observent aussi bien dans la langue que dans les autres formes de création artistique ou artisanale. Un séjour d'un mois à l'université de La Sapienza (Rome) comme «professeur invité » dans le cadre du projet ERC « Tracking Papyrus and Parchment PAThs: An Archaeological Atlas of Coptic Literature » (dir. Paola Buzzi) a été en outre l'occasion de travailler sur les rapports entre livre grec et livre copte.

Un des aspects du multilinguisme écrit est le polygraphisme sur lequel j'ai été maintes fois amené à réfléchir dans le domaine gréco-copte. Le phénomène mérite cependant une approche comparative, raison pour laquelle, avec Marc Smith et Judith Olszowy-Schlanger, j'ai organisé une journée d'étude sur le sujet dans le cadre du groupe transversal en paléographie («Scripta-PSL») qui permette la confrontation des diverses modalités du polygraphisme selon les aires culturelles («Polygraphisme : écritures en coexistence et en interférence », Collège de France, le 6 avril 2018). Le sujet donnera lieu, du 28 février au $1^{\text {er }}$ mars 2019, à un colloque international du programme IRIS «Scripta-PSL. Histoire et pratiques de l'écrit» (dont je dirige l'axe « Pratiques documentaires, anciennes et modernes »).

L'autre volet de mes recherches s'organise autour de l'écrit documentaire auquel j'essaie d'appliquer les mêmes outils d'analyse qu'à l'écrit littéraire dans la mesure où la question du rapport de la forme et du sens se pose aussi pour ces types d'écrit et où, à partir du III ${ }^{\mathrm{e}}$ siècle après J.-C., le premier est de plus en plus influencé par les normes de l'écrit littéraire. Cela m'amène à analyser le document comme un objet culturel et non pas seulement comme le véhicule d'un contenu. La série de cours que j'ai donnés à Florence en mai 2018 («Il documento : un nuovo sguardo sulla cultura scritta della tarda Antichità ») a tenté de synthétiser les diverses directions de cette recherche de longue haleine qui se concrétisera sous la forme d'un précis de poétique documentaire dans les papyrus.

Cette démarche comporte évidemment une forte dimension paléographique que j'ai pu développer dans une communication et une conférence consacrées, pour l'une, aux papyrus grecs ( Formats and scripts as signifiers of Late Antique written culture: Some considerations », Workshop «Text-Object-Person (TOP) », université d'Heidelberg, 26-28 juin 2018), pour l'autre, aux papyrus coptes («Coptic 
documentary palaeography: How to describe a handwriting? and what purpose for palaeography? », 6th Summer School of Coptic Papyrology, Paris, 2-11 juillet 2018).

L'édition de textes inédits a continué à être au centre de mes activités : outre l'édition de papyrus isolés (notamment un petit ensemble de reçus de convoiement de l'annone par le monastère de la Métanoia, en cours d'édition), j'ai lancé une double entreprise éditoriale : la publication d'un nouveau volume de papyrus de la collection Fouad (IFAO, Le Caire) - qui a donné lieu à une mission sur place en compagnie de deux collaborateurs du projet, Nathan Carlig (La Sapienza) et Antonio Ricciardetto (Collège de France) - et d'un volume de papyrus grecs, latins, coptes et arabes de la Bibliothèque Nationale de France.

\section{TRAVAUX DE L'ÉQUIPE}

\section{Collaborateurs}

\section{Antonio Ricciardetto (ATER)}

Antonio Ricciardetto a participé à l'élaboration du site Aphrodito Online ainsi qu'aux projets du professeur, en particulier celui d'édition et de réédition de papyrus grecs conservés à la Bibliothèque nationale de France et à l'Institut français d'archéologie orientale du Caire. Dans ce cadre, il a effectué un séjour de recherches à l'IFAO (22 janvier-12 février 2018). Il a aussi collaboré à la préparation de colloques internationaux et de journées d'études organisés par le professeur contribuant à la mise en forme de deux volumes d'actes (Civilisations en transition. (IV). Patrimoine partagé et patrimoine identitaire, Byblos, 2018, et Curiosité d'Égypte. Entre quête de soi et découverte de l'autre, à paraître dans la collection « Hautes études du monde gréco-romain », Genève).

Antonio Ricciardetto a poursuivi ses recherches sur la médecine dans l'Égypte gréco-romaine, dont il a exposé les principaux résultats lors de manifestations scientifiques à Berlin (13 novembre 2017), Naples (5 décembre 2017), Liège (14 et 21 décembre 2017 ; 4 juin 2018), Paris (13 juin 2018) et Oslo (24 juin 2018). Il a aussi été le réviseur du volume de J. Jouanna, Hippocrate. Serment, Serments chrétiens, Loi (Paris, Les Belles Lettres, CUF, 2018). En tant que secrétaire de la Société belge d'études byzantines, il a organisé la $17^{\mathrm{e}}$ Journée d'études byzantines (Gand, 25 mai 2018). Il est aussi le coorganisateur, avec Á. Mihailyko et N. Korvoll, du workshop « Healing, Belief and Placebo: Medical and Religious Plurality in Late Antiquity » (Oslo, 24-26 juin 2018).

Publications :

RICCIARDETTO A., «Les références à la maladie et à son traitement dans les archives de Zénon », Histoire des sciences médicales, vol. 51, n 2, 2017, p. 153-162.

RICCIARDETTO A., « Spazio scritto e spazio non scritto nelle dossografie mediche su papiro », in N. Pellé (dir.), Spazio scritto e spazio non scritto nel libro papiraceo : esperienze a confronto. Atti della $2^{a}$ tavola-rotonda del Centro di Studi Papirologici, Lecce, Pensa Multimedia, coll. «Edaphos », nº 2, 2017, p. 183-224.

RICCIARDETTO A., « Témoignages sur l'art vétérinaire dans les lettres conservées sur papyrus, sur ostraca et sur tablettes (III ${ }^{\mathrm{e}} \mathrm{s}$. avant notre ère-VII ${ }^{\mathrm{e}} \mathrm{s}$. de notre ère) », in A.-M. DOYENHIGUET et B. VAN DEN ABEELE (dir.), Chevaux, chiens, faucons : l'art vétérinaire antique et médiéval à travers les sources écrites, archéologiques et iconographiques, Louvain-la-Neuve, 
Université catholique de Louvain, coll. «Publications de l'Institut d'études médiévales: Textes, études, congrès », no 28, 2018, p. 91-108.

RICCIARDETTO A., « P.Leod. inv. 2 : fragment d'un document relatif à un bien immobilier ( ?)

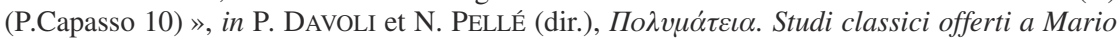
Capasso, Lecce, Pensa Multimedia, 2018, p. 81-88.

Ricciardetto A. et Gourevitch D., «Moi, Téreus, enceinte et battue », La revue du praticien, vol. 68, n 6, 2018, p. 690-694.

RICCIARDETTO A., «L'utilisation thérapeutique et cosmétique des produits tirés des crocodiles dans l'antiquité gréco-romaine », in V. BOUdON-Millot et M. PARDON-LABONNELIE (dir.), Le teint de Phrynè. Thérapeutique et cosmétique dans l'antiquité, Paris, De Boccard, coll. « Orient \& Méditerranée », nº 27, 2018, p. 51-75.

\section{Loreleï Vanderheyden (ATER, également rattachée à la chaire de N. Grimal)}

Loreleï Vanderheyden a poursuivi sa participation à la mise en œuvre du site Aphrodito Online et a collaboré à l'organisation du colloque et des journées d'études du professeur qui se sont tenus au Collège de France. Elle a présenté une communication à Lausanne le 20 octobre 2017 sur l'administration des monastères à la lumière des papyrus grecs et coptes d'Aphrodité ( $\mathrm{VI}^{\mathrm{e}}-\mathrm{VIII} \mathrm{e}^{\mathrm{e}}$ siècles) et soumis deux articles dans le domaine des études coptes. Elle a enfin encodé des textes coptes dans la Duke Database of Documentary Papyri contribuant ainsi à une meilleure visibilité de la documentation copte et a continué la veille documentaire des «Éditions et études papyrologiques disponibles en libre accès sur internet» accessible depuis la page recherche du professeur sur le site du Collège de France. Son activité au Collège s'est interrompue plus tôt que prévu à la suite de son recrutement par l'université de Rome-La Sapienza en tant que chercheuse postdoctorale sur le programme ERC NOTAE (NOT A writtEn word but graphic symbols. NOTAE: An evidence-based reconstruction of another written world in pragmatic literacy from Late Antiquity to early medieval Europe).

\section{L'équipe « Monde byzantin » (UMR 8167)}

Les chercheurs de l'équipe Monde Byzantin (UMR 8167) ont continué les entreprises déjà lancées :

- archives de l'Athos: volumes sur les archives des monastères de Vatopédi, Zographou (monastère bulgare) et Chilandar (monastère serbe) ;

- archives de l'Athos: catalogue des manuscrits grecs du monastère de Zographou dans le cadre du projet européen Athoniki Psiphiaki Kibotos ;

- séminaire paléologue sur «Byzance après 1204 », permettant un échange régulier sur les nombreuses recherches en cours sur cette période, en France et à l'étranger ;

- l'énorme travail d'édition du De cerimoniis, le traité recensant le protocole impérial du $\mathrm{X}^{\mathrm{e}}$ siècle, touche à sa fin ;

- l'unité coorganise le séminaire «Imago-Eikon. Regards croisés sur l'image chrétienne médiévale entre Orient et Occident»;

- les archéologues ont d'une part poursuivi les recherches sur l'Europe centrale et orientale au Moyen Âge, d'autre part sur l'Albanie et les Balkans; la présence d'une chercheuse du service archéologique syrien, W. Khoury, a permis de prolonger d'une autre manière les travaux antérieurs sur la Syrie, zone d'une richesse 
exceptionnelle; l'inventaire archéologique de l'Italie du Sud continue, avec le monastère de Fragala ;

- le programme «Les mobilités monastiques en Orient et en Occident de l'Antiquité tardive au Moyen Âge (IV $-X^{\mathrm{e}}$ siècle) », qui s'achève et dont les deux colloques de Rome et de Vienne sont à paraitre en 2019 à l'EfR (manuscrit déposé, collaboration entre Monde byzantin, EfR, IFAO et Labex RESMED).

- collaboration poursuivie avec l'Académie des sciences de Vienne (M. Popovic et al.) autour du programme de cartographie en ligne : «Bithynia DPP - Mapping the French surveys on line. The Byzantine Region of Bithynia (4th-15th c.) » (avec le Labex Resmed).

\section{Publications :}

BLANCHET M.-H., «L'usage de la censure dans l'exercice du pouvoir impérial à l'époque paléologue : la politique ecclésiastique », in B. CASEAU, A. SOPRACASA et V. PRIGENT (dir.),

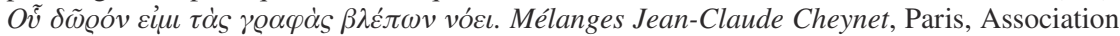
des amis du Centre d'histoire et civilisation de Byzance, coll. "Travaux et Mémoires », vol. 21, $\mathrm{n}^{\mathrm{o}}$ 1, 2017, p. 21-30.

BLANCHET M.-H., «Une acolouthie inédite pour la réconciliation des apostats attribuée au patriarche Gennadios II : Édition princeps et commentaire », in C. GASTGEBER, E. MITSIOU, J. PREISER-KAPELLER et V. ZERVAN (dir.), The patriarchate of Constantinople in context and comparison. Proceedings of the International Conference, Vienna, september 12th-15th 2012. In Memoriam Konstantinos Pitsakis 1944-2012 and Andreas Schminck 1947-2015, Vienne, Verlag der Österreichischen Akademie der Wissenschaften, coll. «Denkschriften der philosophisch-historischen Klasse », n 502, 2017, p. 183-196.

Brodbeck S., De Giorgi M. et Jolivet-Lévy C., «La decorazione pittorica : stato di conservazione e descrizione analitica del programma pittorico », in S. BRODBECK, M. DE Giorgi, M. Falla Castelfranchi, C. Jolivet-Lévy et M.-P. Raynaud (dir.), San Filippo di Fragalà, monastero greco delle Sicilia normanna $=$ San Filippo de Fragalà : monastère grec de la Sicile normande, Rome/Bari, École française de Rome/Mario Adda Editore, «Collection de l'École française de Rome », nº 553, 2018, p. 83-158.

BRodbeck S. et Jolivet-LÉVY C., «Les peintures de Fragalà, Byzance et le premier art siculo-normand », in S. BRodBeCK, M. De Giorgi, M. FAlla CASTELFRANCHI, C. JolivetLÉVY et M.-P. RAYNAUd (dir.), San Filippo di Fragalà, monastero greco delle Sicilia normanna $=$ San Filippo de Fragalà : monastère grec de la Sicile normande, Rome/Bari, École française de Rome/Mario Adda Editore, "Collection de l'École française de Rome », $\mathrm{n}^{\mathrm{0}} 553,2018$, p. $183-204$.

Brodbeck S., De Giorgi M., Falla Castelfranchi M., Jolivet-Lévy C. et RAYNAUD M.-P. (dir.), San Filippo di Fragalà, monastero greco delle Sicilia normanna = San Filippo de Fragalà : monastère grec de la Sicile normande, Rome/Bari, École française de Rome/Mario Adda Editore, «Collection de l'École française de Rome », nº 553, 2018.

CASEAU B., "L'exercice de la charité à Byzance d'après les sceaux et les tessères

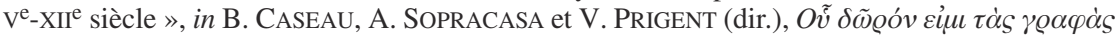
$\beta \lambda \varepsilon ́ \pi \omega v$ vóel. Mélanges Jean-Claude Cheynet, Paris, Association des amis du Centre d'histoire et civilisation de Byzance, coll. «Travaux et Mémoires », vol. 21, nº 1, 2017, p. 31-52.

CASEAU B., «Les Byzantins en mission », Historia, hors-série no 7 : Une histoire du Liban, des Phéniciens à nos jours, Paris, Sophia Publications, 2017, p. 26-27.

CASEAU B. et FAYANT M.-C., « Le renouveau du culte des stylites syriens aux $\mathrm{X}^{\mathrm{e}}$ et $\mathrm{XI}^{\mathrm{e}}$ siècles ? La Vie abrégée de Syméon Stylite le Jeune (BHG 1691c)», in J.-C. CheYNET et B. Flusin (dir.), Autour du Premier humanisme byzantin \& des Cinq études sur le $\mathrm{XI}^{\mathrm{e}}$ siècle, 
quarante ans après Paul Lemerle, Paris, Association des amis du Centre d'histoire et civilisation de Byzance, coll. «Travaux et Mémoires », vol. 21, n 2, 2017, p. 701-732.

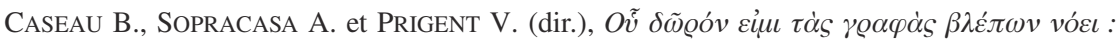
Mélanges Jean-Claude Cheynet, Paris, Association des amis du Centre d'histoire et civilisation de Byzance, coll. « Travaux et Mémoires », vol. 21, nº 1, 2017.

CHEYNET J.-C., « Les impératrices byzantines et leurs réseaux : 1028-1203 », in F. CHAUSSON et S. DESTEPHEN (dir.), Augusta, Regina, Basilissa, La souveraine de l'Empire romain au Moyen Âge. Entre héritages et métamorphoses, Paris, Éditions de Boccard, coll. «De l'archéologie à l'histoire », no 71, 2018, p. 141-158.

Cheynet J.-C., «La société urbaine », in J.-C. Cheynet et B. Flusin (dir.), Autour du Premier humanisme byzantin \& des Cinq études sur le $\mathrm{XI}^{\mathrm{e}}$ siècle, quarante ans après Paul Lemerle, Paris, Association des amis du Centre d'histoire et civilisation de Byzance, coll. « Travaux et Mémoires », vol. 21, nº 2, 2017, p. 449-482.

Cheynet J.-C. et Flusin B. (dir.), Autour du Premier humanisme byzantin \& des Cinq études sur le $\mathrm{XI}^{\mathrm{e}}$ siècle, quarante ans après Paul Lemerle, Paris, Association des amis du Centre d'histoire et civilisation de Byzance, coll. « Travaux et Mémoires », vol. 21, nº 2, 2017.

DELOUIS Olivier, «La Collection canonique du hiéromoine Macaire retrouvée à Orléans (olim Mikulov I 136, nunc Parisinus Suppl. gr. 1394)», in B. CASEAU, V. PRIGENT et

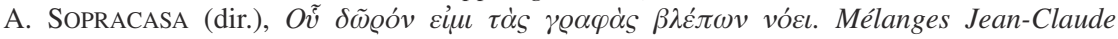
Cheynet, Paris, Association des amis du Centre d'histoire et civilisation de Byzance, coll. « Travaux et Mémoires », vol. 21, nº 1, 2017, p. 67-108.

DÉroche V., « The Doubts about the Saloi in the Middle and Late Byzantine Periods and their Apologetic Reuse in Two Lives of Saloi », in A. BERGER et S.A. IVANOV (dir.), Holy Fools and Divine Madmen : Sacred Insanity through Ages and Cultures, Munich, UG, coll. « Münchner Arbeiten zur Byzantinistik », nº 2, 2018, p. 65-84.

DÉROCHE V., «Des miracles pour la bonne société : la Vie de saint Sampson par Syméon

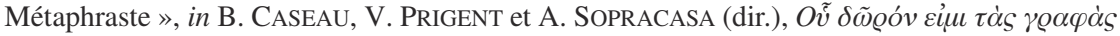
$\beta \lambda \varepsilon ́ \pi \omega v$ vóeı. Mélanges Jean-Claude Cheynet, Paris, Association des amis du Centre d'histoire et civilisation de Byzance, coll. «Travaux et Mémoires », vol. 21, nº 1, 2017, p. 109-122.

DÉROCHE V., «L'autorité religieuse à Byzance», in D. AVON (dir.), Faire autorité. Les religions dans le temps long et face à la modernité, Rennes, Presses universitaires de Rennes, coll. « Histoire », 2017, p. 35-48.

Durand J. (dir.), Le Trésor de Preslav. Reflet d'un âge d'or du Moyen Âge bulgar, Paris, Somogy/ Louvre éditions, 2018.

EstAngÜi Gómez R., « Les premiers exarques du Patriarcat de Constantinople en Macédoine : dernier quart du XIV e siècle », in C. GAstgeber, E. Mitsiou, J. Preiser-Kapeller et V. ZERVAN (dir.), The patriarchate of Constantinople in context and comparison : Proceedings of the International Conference, Vienna, september 12th-15th 2012: In Memoriam Konstantinos Pitsakis 1944-2012 and Andreas Schminck 1947-2015, Vienne, Verlag der Österreichischen Akademie der Wissenschaften, coll. «Denkschriften der philosophischhistorischen Klasse », no 502, 2017, p. 135-160.

FLusin B., «Aréthas de Césarée et la transmission du savoir », in J.-C. CHEYNET et B. Flusin (dir.), Autour du Premier humanisme byzantin \& des Cinq études sur le $\mathrm{XI}^{\mathrm{e}}$ siècle, quarante ans après Paul Lemerle, Paris, Association des amis du Centre d'histoire et civilisation de Byzance, coll. «Travaux et Mémoires », vol. 21, n 2, 2017, p. 309-324.

FLUSIN B., «Remarques sur la date de rédaction du De cerimoniis », in B. CASEAU,

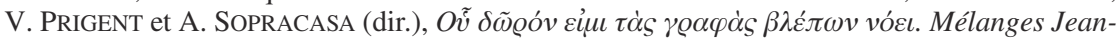
Claude Cheynet, Paris, Association des amis du Centre d'histoire et civilisation de Byzance, coll. « Travaux et Mémoires », vol. 21, nº 1, 2017, p. 151-168. 
GANCHOU T., « La tour d'Irène, Eirene Kulesi, à Istanbul : le palais de Loukas Notaras ? », in

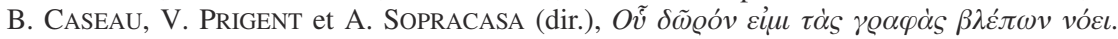
Mélanges Jean-Claude Cheynet, Paris, Association des amis du Centre d'histoire et civilisation de Byzance, coll. « Travaux et Mémoires », vol. 21, nº 1, 2017, p. 169-256.

KAZANSKI M., «Большие «готские пряжки» с изображением креста у населения ЮгоЗападного Крыма (второая половина VI - первая половина VII вв.) : опроисхождении убора [Les grandes plaques-boucles «gothiques » ornées de la croix, la deuxième moitié du $\mathrm{VI}^{\mathrm{e}}$-première moitié du $\mathrm{VII}^{\mathrm{e}}$ siècles: sur l'origine du costume]», in A. MUSIN et O. Sheheglova (dir.), In Stone and Bronze : essays presented in honor of Anna Peskova, Saint-Pétersbourg, Russian Academy of Sciences, 2017, p. 221-226.

Kontouma V., «Christianisme orthodoxe », Annuaire de l'École pratique des hautes études, Section des Sciences religieuses, $\mathrm{n}^{\circ}$ 125, 2018, p. 247-258, https://journals.openedition.org/ asr/2002.

LAZARIS S., «Scientific, medical and technical manuscripts », in V. TSAMAKDA (dir.), A Companion to Byzantine Illustrated Manuscripts, Leyde/Boston, Brill, 2017, p. 55-113, DOI : 10.1163/9789004346239_005.

Morrisson C., «Anglo-Byzantina : monnaies et sceaux outre-Manche IX ${ }^{\mathrm{e}}-\mathrm{XIII}{ }^{\mathrm{e}}$ siècle », in

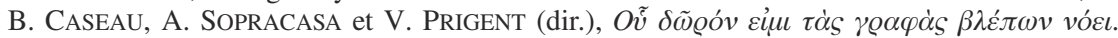
Mélanges Jean-Claude Cheynet, Paris, Association des amis du Centre d'histoire et civilisation de Byzance, coll. « Travaux et Mémoires », vol. 21, nº 1, 2017, p. 471-486.

MORRISSON C., «Revisiter le $\mathrm{XI}^{\mathrm{e}}$ siècle quarante ans après : expansion et crise », in J.-C. Cheynet et B. Flusin (dir.), Autour du Premier humanisme byzantin \& des Cinq études sur le XI siècle, quarante ans après Paul Lemerle, Paris, Association des amis du Centre d'histoire et civilisation de Byzance, coll. «Travaux et Mémoires », vol. 21, $\mathrm{n}^{\mathrm{o}}$ 2, 2017, p. 611-625.

PIERI D., «Les pèlerinages », in R. ZIADÉ (dir.), Chrétiens d'Orient : 2000 ans d'histoire. Exposition présentée à l'Institut du monde arabe, à Paris, du 26 septembre 2017 au 14 janvier 2018, Paris/Tourcoing, Gallimard/Institut du monde arabe/MUba Eugène Leroy, 2017, p. 48-57.

RAPTI I., «Un nouveau témoignage sur l'empire des Grands Comnènes : la date des fresques de la chapelle Saint-Élie au monastère de Vazélon », Revue des études byzantines, vol. 75, 2017, p. 5-39, DOI : 10.2143/REB.75.0.3251286.

SIDÉRIS G., «Les débats sur l'eunucité et la nature physiologique des eunuques à Byzance : $\mathrm{IV}^{\mathrm{e}}-\mathrm{XII}^{\mathrm{e}}$ siècle », in $\mathrm{E}$. PIBIRI et $\mathrm{F}$. АBBOT (dir.), Féminité et masculinité altérées : transgression et inversion des genres au Moyen Âge, Florence, Sismel/Edizioni del Galluzzo, coll. « Micrologus' library », nº 78, 2017, p. 145-206.

Stavrou M., «Une réévaluation du Tomos du Deuxième Concile des Blachernes (1285) : commentaire, tradition textuelle, édition critique et traduction », in C. GASTGEBER, E. Mitsiou, J. PREISER-KAPELlER et V. ZERVAN (dir.), The patriarchate of Constantinople in context and comparison. Proceedings of the International Conference, Vienna, september 12th-15th 2012. In Memoriam Konstantinos Pitsakis [1944-2012] and Andreas Schminck [1947-2015], Vienne, Verlag der Österreichischen Akademie der Wissenschaften, coll. « Denkschriften der philosophisch-historischen Klasse », nº 502, 2017, p. 47-94.

ZIADÉ R., Chrétiens d'Orient : 2000 ans d'histoire. Exposition présentée à l'Institut du monde arabe (Paris, 26 septembre 2017 au 14 janvier 2018) et au MUba Eugène Leroy (Tourcoing, 17 février au 5 juin 2018), Paris/Tourcoing, Gallimard/Institut du monde arabe/MUba Eugène Leroy, 2017.

ZUCKERMAN C., «Appendix : Marinos (PmbZ 4797), count of the Opsikion and exarch of

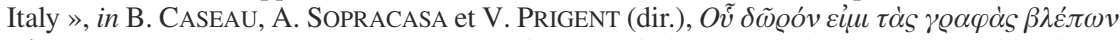
vóel. Mélanges Jean-Claude Cheynet, Paris, Association des amis du Centre d'histoire et civilisation de Byzance, coll. «Travaux et Mémoires », vol. 21, nº 1, 2017, p. 803-806. 
ZuCKERMAN C., «Emperor Theophilos and Theophobos in Three Tenth-Century Chronicles : Discovering the Common Source », Revue des études byzantines, vol. 75, 2017, p. 101-150, DOI : 10.2143/REB.75.0.3251289.

ZUCKERMAN C., «On generals of Armenian origin named Leo in the early 800's, or, The

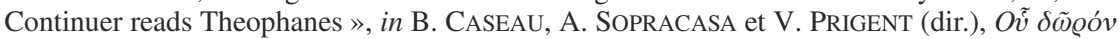

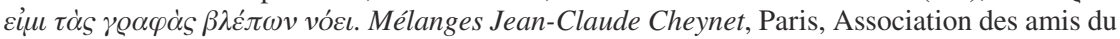
Centre d'histoire et civilisation de Byzance, coll. « Travaux et Mémoires », vol. 21, n 1, 2017, p. 831-849.

\section{Publications}

\section{OUVRAGES}

Fournet J.-L., Mouton J.-M. et PAVIOT J. (dir.), Civilisations en transition (III). Sociétés multiconfessionnelles à travers l'histoire du Proche-Orient. Actes du colloque scientifique international, 7-8-9 septembre 2016, Byblos, Centre international des sciences de 1'homme, 2017.

Fournet J.-L., Mouton J.-M. et PAVIOt J. (dir.), Civilisations en transition (IV). Patrimoine partagé et patrimoine identitaire. Actes du colloque scientifique international, 23-24 octobre 2017, Byblos, Centre international des sciences de l'homme, 2018.

\section{ARTICLES OU CONTRIBUTIONS À DES OUVRAGES}

FOURnET J.-L., « Tensions religieuses dans l'Égypte de l'Antiquité tardive », in J.-L. FourNET, J.-M. Mouton et J. PAVIOT (dir.), Civilisations en transition (III). Sociétés multiconfessionnelles à travers l'histoire du Proche-Orient, Byblos, Centre international des sciences de l'homme, 2017, p. 63-92.

Fournet J.-L., «Compte rendu de Lincoln H. Blumell et Thomas A. Wayment, Christian Oxyrhynchus: texts, documents, and sources, Waco, Baylor University Press, 2015, 756 p., ISBN: 9781602585393 », Archiv für Papyrusforschung und verwandte Gebiete, vol. 63, n 2, 2017, p. 415-420, DOI : 10.1515/apf-2017-0034.

FOURNET J.-L., «'Alessandria laboratorio di ogni forma di cultura' : la vita universitaria ad Alessandria nella tarda antichità », in S. Russo (dir.), Santa Catarina d'Egitto. L'Egitto di Santa Catarina, Florence, Edizioni Polistampa, 2017, p. 29-43.

FOURNET J.-L., «Les documents bilingues gréco-coptes dans l'Égypte byzantine : essai de typologie », in M. LAFKIOUI et V. BRUGNATELLI (dir.), Written sources about Africa and their study. Le fonti scritte sull'Africa e $i$ loro studi, Milan, Biblioteca Ambrosiana/Centro Ambrosiano, coll. «Africana Ambrosiana », no 3, 2018, p. 59-83.

FOURNET J.-L., «Archives and libraries in Greco-Roman Egypt», in A. BAUSI, C. BROCKMANN, M. FrIEDRICH et S. KIENITZ (dir.), Manuscripts and Archives : Comparative Views on Record-Keeping, Berlin/Boston, De Gruyter, 2018, p. 171-200, DOI : $10.1515 / 9783110541397$.

FOURnet J.-L., «Le désert Oriental dans l'Antiquité tardive », in J.-P. BRUN, T. FAUCHER, B. Redon et S. Sidebotham (dir.), Le désert Oriental d'Égypte durant la période grécoromaine : bilans archéologiques, Paris, Collège de France, coll. «Institut des civilisations », 2018, DOI : 10.4000/books.cdf.5187.

Fournet J.-L., «The Eastern Desert in Late Antiquity », in J.-P. Brun, T. FAUCHER, B. Redon et S. Sidebotham (dir.), The Eastern Desert of Egypt during the Greco-Roman Period: Archaeological Reports, Paris, Collège de France, coll. «Institut des civilisations », 2018, DOI : 10.4000/books.cdf.5230. 
FOURNET J.-L., « Culture écrite de l'Antiquité tardive et papyrologie byzantine », Annuaire du Collège de France 2015-2016. Résumé des cours et travaux, 116 a année, Paris, Éditions du Collège de France, 2018, p. 261-280, DOI : 10.4000/annuaire-cdf.12873.

FOURNET J.-L., « Changements religieux et patrimoine cultuel : le sort des temples égyptiens durant l'Antiquité tardive », in J.-L. Fournet, J.-M. Mouton et J. PAVIOT (dir.), Civilisations en transition (IV). Patrimoine partagé et patrimoine identitaire, Byblos, Centre international des sciences de l'homme, 2018, p. 45-81.

FOURNET J.-L., « Le faux en écriture d'après la documentation papyrologique », in H. GABER, N. GRIMAL et O. PERDU (dir.), Imitations, copies et faux dans les domaines pharaonique et de l'Orient ancien, Paris, Aibl/Soleb, coll. «Études d'égyptologie», no 16, 2018, p. 274-303.

FOURNET J.-L., «Sur les premiers documents juridiques coptes (3) : Les "archives" d'Apa Abraham », in A. Boud'HORS et C. LouIS (dir.), Études Coptes XV. Dix-septième journée d'études, Lisbonne, 18-20 juin 2015, Paris, De Boccard, coll. «Cahiers de la bibliothèque copte $», n^{0} 22,2018$, p. 199-226.

FOURNET J.-L., «La papyrologie », in P. HENRIET (dir.), L'École pratique des hautes études : invention, érudition, innovation de 1868 à nos jours, Paris, École pratique des hautes études/ Somogy éditions d'art, 2018, p. 336-339. 